\title{
The constrained space orbital variation analysis for periodic ab initio calculations
}

\author{
N. Cruz Hernández ${ }^{\mathrm{a})}$ and Claudio Marcelo Zicovich-Wilson \\ Departamento de Física, Facultad de Ciencias, Universidad Autónoma del Estado de Morelos, Avenida \\ Universidad 1001, Col. Chamilpa, Cuernavaca, Morelos 62210, México \\ Javier Fdez. Sanz ${ }^{\text {b) }}$ \\ Departamento de Química Física, Facultad de Química, Universidad de Sevilla, E-41012 Sevilla, Spain
}

(Received 16 February 2006; accepted 29 March 2006; published online 16 May 2006)

\begin{abstract}
The constrained space orbital variation (CSOV) method for the analysis of the interaction energy has been implemented in the periodic ab initio CRYSTAL03 code. The method allows for the partition of the energy of two interacting chemical entities, represented in turn by periodic models, into contributions which account for electrostatic effects, mutual polarization and charge transfer. The implementation permits one to carry out the analysis both at the Hartree-Fock and density functional theory levels, where in the latter the most popular exchange-correlation functionals can be used. As an illustrating example, the analysis of the interaction between $\mathrm{CO}$ and the $\mathrm{MgO}(001)$ surface has been considered. As expected by the almost fully ionic character of the support, our periodic CSOV results, in general agree with those previously obtained using the embedded cluster approach, showing the reliability of the present implementation. () 2006 American Institute of Physics.
\end{abstract} [DOI: $10.1063 / 1.2198528$ ]

\section{INTRODUCTION}

The decomposition of the interaction energy between two chemical entities on the grounds of the underlying physical contributions constitutes one of the main issues in the analysis and understanding of chemical interacting systems. With this purpose, both perturbational and variational computational procedures have been proposed. In both cases, the interaction energy is rebuilt by computing different terms from the wave functions of the separate monomers.

The constrained space orbital variation (CSOV) method was initially introduced by Bagus et al. ${ }^{1,2}$ and falls in the framework of the variational type of approaches, first developed by Morokuma ${ }^{3}$ and Ziegler and Rauk. ${ }^{4}$ This procedure allows one to decompose the interaction energy into physically meaningful contributions, building specific variational spaces on the basis of the orbitals of isolated monomers. Depending on the set of orbitals frozen, it is then possible to estimate the different physical contributions to the interaction energy. Although the CSOV method was originally intended for the analysis of the interaction of closed shell ligands with metals, it was later generalized and can be applied to decompose the interaction energy of almost any chemical bond..$^{5}$ In particular, it has been extensively used to analyze the interaction between adsorbates and surfaces.

As most of the variational procedures for analysis, the CSOV method was initially implemented on a Hartree-Fock (HF) based scheme and most of its applications have been performed at such uncorrelated level. Nevertheless, the influ-

\footnotetext{
${ }^{a)}$ On leave from Departamento de Química Física, Universidad de Sevilla, Spain.

b) Author to whom correspondence should be addressed. Fax: +34954557177. Electronic mail: sanz@us.es
}

ence that electron correlation exerts on the adsorbatesubstrate interaction energy suggests that the contribution of the various physical mechanisms involved in a given chemisorption bond is also susceptible to change. However, decomposing the interaction energy obtained from highly correlated $a b$ initio wave functions appears to be a quite involved task, which is only achieved for some simple multiconfiguration self-consistent-field (MCSCF) cases. ${ }^{6}$ This limitation was later overcome using the single determinant form of the Kohn-Sham (KS) approach to density functional theory (DFT) which allows one to perform the CSOV analysis in a rather straightforward way simply using the KS orbitals in the variational procedure. This implementation was first done by Neyman and co-workers ${ }^{7,8}$ and later incorporated in a general purpose program as HONDO95 by Dupuis et $a l .{ }^{9}$ A comparison of the differences between the HF and the DFT physical contributions to the interaction energy has also been reported. ${ }^{10}$

A still remaining drawback of the CSOV method arises from its original design oriented to molecular species, and, therefore, its application is limited to "molecular" cases, i.e., gas phase calculations or extended systems described through finite models. As mentioned above the method has been extensively used to interpret adsorbate-surface interactions, in which case the surface has been modeled by using either finite clusters or clusters embedded in an array of point charges and/or model potentials to at least incorporate into the electronic Hamiltonian the electrostatic contributions of the environment (see for instance Ref. 11). This, of course, limits the application of the method to those materials where such embedded cluster models are possible and even then 
one have to deal with the actual value of the point charges, an issue which is always difficult to set up for any not fully ionic material. ${ }^{12}$

The technical limitations of the ab initio embedded cluster models in surface chemistry and chemisorption are clearly overcome by the use of ab initio theory under periodic boundary conditions. Either using plane waves or Gaussian functions as basis set, this formulation allows for an elegant and powerful description of the extended nature of a solid employing an infinite model. ${ }^{13,14}$ Because of its computational advantages and reliability, the periodic ab initio theory has been widely used during the last years, up to the point that most of the theoretical research on surface properties published nowadays makes use of periodic codes. Nevertheless, the analysis and physical interpretation of the surface-adsorbate interaction can only be made at a qualitative level in many cases. For example, the study of the charge transfer between a supported metal and a surface can qualitatively be performed from the maps of the electron density differences, but to obtain quantitative conclusions it is necessary to integrate the density for a given (unknown) radius. More details might also be obtained from the density of states projected onto some atoms and by analyzing the new features with respect to the isolated system. However, a quantitative conclusion and a direct estimation of such contributions to the interaction energy are still not possible.

As a practical alternative to circumvent these issues there still is the possibility to use mixed periodic-cluster strategies. In this case the periodic based calculations are followed by an analysis using a cluster tailored from the infinite model. This alternative has recently been used, for instance, in the analysis of the interaction energy between alumina surfaces and transition metals as $\mathrm{Cu}, \mathrm{Ag}, \mathrm{Au}$, and Pd. ${ }^{15,16}$ However, besides its unwieldiness, a key aspect in such a type of calculations refers to the surface relaxation that can change not only the energetics of the problem, but also the qualitative description of the chemisorption, ${ }^{17}$ and that it is not always properly accounted for in embedded cluster calculations.

In this paper, we present for first time the combination of the advantages of periodic model and CSOV analysis. To this purpose we have extended the CSOV strategy to account for the crystalline systems. In this scheme the partition into variational spaces is not performed on the whole model space, which in the periodic model is infinite, but on a set of subspaces invariant under space symmetry operators. Owing to the computational techniques usually employed in the periodic approach, those subspaces are of finite dimension, and only a finite number of them are enough to describe the whole system. The procedure has been implemented in the periodic code CRYSTAL03. ${ }^{18}$ Beyond the well-known reliability and performance of the code, the choice provides, in this case, several additional advantages with respect to the most popular plane-wave methods. First of all, CRYSTAL spans the wave function as a linear combination of atomic orbitals, being therefore a natural extension of the procedures used in traditional quantum chemistry into which the CSOV analysis has been formulated. In addition, the full exploitation of space symmetry performed in the code provides not only a better performance in many symmetric surface-adsorbate systems, but also allows for an efficiently use of the CSOV scheme in the study of the interaction between general threedimensional (3D) sublattices, a situation that often occurs in clathrates, mixed molecular crystals, or zeolite chemistry, among others. Last but not the least, the periodic theory under localized basis sets permits to easily and efficiently compute the exact exchange energy. Beyond its usefulness in the computation of the HF energy, the exact exchange allows to define hybrid DFT exchange functionals that are widely employed in the molecular quantum chemistry owing to its very often revealed accuracy, in many cases comparable to high level correlation methods but at signficantly lower computational cost.

The paper is arranged as follows. In the next section, after a brief description of the CSOV method, we report the basic aspects of the implementation in a periodic model. In Sec. III we perform a test of the procedure using as a case study the well-known problem of adsorption of $\mathrm{CO}$ on the $\mathrm{MgO}(001)$ surface. This system has been extensively studied in the past years and may be considered as a paradigm in surface science which has been the subject of many theoretical papers, ${ }^{19}$ not always exempt of controversy (see for instance Refs. 20 and 21 and references therein). Finally the main conclusions are outlined in Sec. IV.

\section{THE CSOV ANALYSIS FOR PERIODIC SYSTEMS}

\section{A. The CSOV method}

The CSOV foundations have been described in detail in the literature (see for instance Ref. 5), however, to properly understand the physical significance of the periodic implementation it is necessary to briefly review the different steps that are followed in the molecular case. In the CSOV analysis, the interaction between two fragments or units $A$ and $B$ is analyzed by computing wave functions where variational changes from the initial separated fragments are allowed to occur in well-defined, controlled steps. Each step measures a physically interpretable contribution to the studied observable in such a way that it is decomposed into a nonbonding contribution (Pauli or nonbonding electronic repulsion) plus bonding contributions corresponding to internal (intraunit) rehybridization or polarization and (interunit) charge transfer contributions. To set up the procedure it is first necessary to divide the full variational space of the supersystem $A B$ to form sets of orbitals that will be used in the constrained variations. The full variational space is divided into four sets. The occupied orbitals on $A$ and $B$ form two of these sets, denoted by $O A$ and $O B$, and the unoccupied, or virtual, orbitals on $A$ and $B$ form the other two sets, denoted by $V A$ and $V B$. The starting point of this process, step number 0 , corresponds to the simple superposition of the separated frozen densities $(\mathrm{FO}=$ frozen orbital) of both fragments. At this step no variation or changes in the superposed charge density other than proper normalization of the FO wave function for $A B$ are allowed. Only the electrostatic interactions between $A$ and $B$ are considered for this starting point; this includes the Pauli repulsion due to the nonbonding overlap or interpenetration of the charge distributions $O A$ and $O B$. In the next 
step, the fragment $A$ electron density is fixed, but the orbitals arising from fragment $B$ are allowed to vary in their own basis space by adding the set $V B$ to the variational space (which of course must be kept orthogonal to that of $A$, yielding the $B$ polarization contribution to the observable. In CSOV step 2, the $A$ orbitals are still fixed, but now the $B$ orbitals are varied in a space that includes the virtual unoccupied molecular orbitals of A. This measures the effect of $B$ to $A$ charge transfer and covalent bonding, and unfortunately, it also accounts for a possible basis set superposition error (BSSE). In a similar way, in CSOV step 3, the $A$ fragment is relaxed in its virtual space adding the set $V A$ to the varational space (while the occupied space of $B$ is that obtained at step 2 , the virtual space of $B$ is restored at its initial configuration, and the whole set is properly orthogonalized), giving the $A$ polarization contribution. Finally, in CSOV step 4, the $A$ to $B$ charge donation contribution is measured. The difference between the sum of all these contributions and the full variational, unconstrained result yields an estimation of the completeness of the variational freedom allowed to the wave function during the CSOV procedure, so that small values of this difference indicate that essentially all important bonding effects have been taken into account during the CSOV analysis.

\section{B. Periodic implementation}

The basic idea of the present method is to exploit the diagonal block factorization of the Fock (and KS) matrix when it is given in terms of a basis of symmetry adapted orbitals. Since orbitals belonging to different irreducible subspaces do not mix during any variational procedure, the CSOV analysis can be performed independently in each subspace without any loss in the description, while giving the possibility to deal with infinite periodic systems, as it is shown in what follows.

According to the previous considerations, let us now consider a crystalline system, that for convenience we will also denote as $A B$, although now it refers to the interaction between two component sublattices, namely, $A$ and $B$. All three systems have the same periodicity, therefore the interaction energy per unit cell between both sublattices reads $\Delta E_{A B}=E_{A B}-E_{A}-E_{B}$, where the terms at the right hand side are the total energy per unit cell of the full and the component lattices, respectively. To perform a quantitative evaluation of the weight of the different physicochemical effects involved in the interaction between $A$ and $B, \Delta E_{A B}$, we follow a way parallel to that of CSOV for molecules and we start by considering the partition of the Fock (or KS) matrix of the whole system $A B$ into sub-blocks, attributed to occupied and virtual orbitals of each component sublattice. This is performed via a previous transformation into a basis set constituted by the re-orthonormalized canonical orbitals of $A$ and $B$ computed separately.

In CRYSTAL (Ref. 22) a basis set of symmetry adapted crystalline orbitals ${ }^{23}$ (SACOs) is used to transform the Fock matrix into block diagonal form, each block being in one-toone correspondence to an irreducible representation (irrep) of the space symmetry group of the system. These irreps are labeled here by the symbol $(\mathbf{k}, r)$, which refers to point $\mathbf{k}$ in the Brillouin Zone (BZ) of reciprocal space and the $r$-irreducible representation of the corresponding $\mathbf{k}$-little group, respectively. Each SACO is a linear combination of $\mathbf{k}$-atomic Bloch functions generated from a translationally invariant set of localized atomic orbitals $\left\{\varphi_{\mu}(\mathbf{r})\right\}_{\mu=1}^{N}$ according to

$$
\varphi_{\mu}^{\mathbf{k}}(\mathbf{r})=\sum_{\mathbf{g}} e^{i \mathbf{k} \cdot \mathbf{g}} \varphi_{\mu}\left(\mathbf{r}-\mathbf{s}_{\mu}-\mathbf{g}\right),
$$

where $\mathbf{s}_{\mu}$ gives the position in the reference cell of the nucleus on which orbital $\mu$ is centered, and the sum runs over the set of lattice vectors. Atomic orbitals are in turn expressed as linear combinations of Gaussian-type orbitals such as in most molecular quantum chemistry implementations. Concerning the set of $\mathbf{k}$ points considered for sampling along the BZ, it is finite and generated employing the widely used Monkhorst-Pack scheme. ${ }^{24}$ Each block of the Fock matrix in the SACO basis set, $F_{i j}^{(\mathbf{k}, r)}$, is independently diagonalized during the SCF procedure to compute the corresponding occupied and virtual crystalline orbitals and energy bands.

In the present periodic CSOV implementation, the symmetry of the overall system $A B$ is also imposed on the component sublattices $A$ and $B$. This permits one to straightforwardly extend the CSOV partition scheme, initially formulated for a single molecular Fock matrix, to each $(\mathbf{k}, r)$ block in the periodic case. In this approach the SACOs used to construct the Fock matrix blocks of $A B$ are actually the canonical Bloch orbitals of $A$ and $B$ (already symmetry adapted). At each $(\mathbf{k}, r)$ irrep there are $n_{(\mathbf{k}, r)}$ occupied and $p_{(\mathbf{k}, r)}$ virtual orbitals in $A$, as well as $m_{(\mathbf{k}, r)}$ occupied and $q_{(\mathbf{k}, r)}$ virtual orbitals in $B$, which span subspaces $O A_{(\mathbf{k}, r)}, V A_{(\mathbf{k}, r)}$, $O B_{(\mathbf{k}, r)}$, and $V B_{(\mathbf{k}, r)}$, respectively. The set of orbitals is next Gram-Schmidt orthonormalized and employed to construct a transformation matrix, namely, $\mathbf{T}^{(\mathbf{k}, r)}$, which allows to compute a new $(\mathbf{k}, r)$-Fock matrix according to

$$
\mathbf{F}^{\prime(\mathbf{k}, r)}=\left(\mathbf{T}^{(\mathbf{k}, r)}\right)^{+} \mathbf{F}^{(\mathbf{k}, r)} \mathbf{T}^{(\mathbf{k}, r)} .
$$

Following the original proposal, ${ }^{1}$ this periodic CSOV implementation is developed in several steps. In each of them, a set of $\mathbf{F}^{\prime(\mathbf{k}, r)}$ matrices is generated by means of transformations $\mathbf{T}^{(\mathbf{k}, r)}$. In these matrices some of the sub-blocks that correspond to the coupling of a pair of subspaces $P$ and $Q$, namely, $\mathbf{F}_{P, Q}^{\prime(\mathbf{k}, r)}$, where $P, Q \in\left\{O A^{(\mathbf{k}, r)}, O B^{(\mathbf{k}, r)}, V B^{(\mathbf{k}, r)}, V A^{(\mathbf{k}, r)}\right\}$ set to zero, and the modified $(\mathbf{k}, r)$-Fock matrices are used to initiate the constrained SCF calculations. The steps are as follows:

- Step 0: matrices $\mathbf{T}^{(\mathbf{k}, r)}$ are constructed for each irrep by orthonormalizing the eigenvectors of $A$ and $B$ in the order $O A^{(\mathbf{k}, r)} \oplus O B^{(\mathbf{k}, r)} \oplus V B^{(\mathbf{k}, r)} \oplus V A^{(\mathbf{k}, r)}$. In the resulting $\mathbf{F}^{\prime(\mathbf{k}, r)}$ matrices, all but the diagonal sub-blocks are set to zero. In this way, orbital variation inside each subspace is only allowed.

- Step 1: The eigenvectors of the previous step are now used to construct matrices $\mathbf{T}^{(\mathbf{k}, r)}$ and $\mathbf{F}^{\prime(\mathbf{k}, r)}$. The mixing between occupied and virtual orbitals of $B$ under the 
field generated by $A$ is now allowed by including $\mathbf{F}_{O B, V B}^{\prime(\mathbf{k}, r)}$ among the nonzero sub-blocks.

- Step 2: As in step 1, the transformed Fock matrices are computed using the eigenvectors of the previous step. In this case the variation space is extended making zero the same sub-blocks as in step 1 except for $\mathbf{F}_{O B, V B}^{\prime(\mathbf{k}, r)}$.

- Step 3: Matrices $\mathbf{T}^{(\mathbf{k}, r)}$ are reconstructed at this step like in the case of step 0 , considering now the order $O A^{(\mathbf{k}, r)} \oplus O B^{(\mathbf{k}, r)} \oplus V A^{(\mathbf{k}, r)} \oplus V B^{(\mathbf{k}, r)}$ for the GramSchmidt orthonormalization and the occupied orbitals after step 2 as basis sets for subspaces $O A^{(\mathbf{k}, r)}$ and $O B^{(\mathbf{k}, r)}{ }^{25}$ All sub-blocks of the initial Fock matrix blocks excepting the diagonal ones and $\mathbf{F}_{O A, V A}^{\prime(\mathbf{k}, r)}$ are set to zero so as to initiate the SCF process.

- Step 4: Similar to step 2, but exchanging $A$ and $B$.

- Step 5: All blocks are kept from the original $\mathbf{F}^{\prime(\mathbf{k}, r)}$ block matrices. A nonconstrained orbital variation is performed in the SCF.

The CSOV energy partition of the interaction energy is given by five terms $\Delta E(n)=E(n)-E(n-1), n=1, \ldots, 5$, where $E(n)$ is the total energy obtained at the $n$th CSOV step.

\section{TESTING THE CSOV IMPLEMENTATION}

Many systems of the form atom/molecule deposited on a surface have been analyzed in order to understand the properties of the adsorbate-substrate bond. The (001) surface of $\mathrm{MgO}$ is certainly one of these "ideal surfaces," for which a vast collection of both experimental and computational modeling results have been reported. Since carbon monoxide has been widely used as probe molecule in surface science, the $\mathrm{CO} / \mathrm{MgO}$ (001) system constitutes the prototypical case study specially suited to test our implementation. Indeed, many theoretical studies, with different degrees of sophistication, have addressed such an interaction. ${ }^{19,26,27}$ Nevertheless, although the system seems to be structurally simple, the accurate estimation of the interaction energy appears to be rather elusive since the binding energy (BE) is actually very small: $0.13 \mathrm{eV}$ experimentally determined using a $\mathrm{MgO}$ single crystal. ${ }^{28}$

A theoretical analysis of the nature of the interaction between $\mathrm{CO}$ and the perfect (001) surface was first reported by Pacchioni et $a .^{29}$ employing an embedded cluster approach. Using the CSOV partition scheme these authors concluded that electrostatic and Pauli exchange repulsion were the main driving forces, whereas charge transfer was negligible. Somewhat later Mejías et al. ${ }^{11}$ reported a detailed analysis of this interaction using clusters of different size embedded in both an array of point charges and model potentials. Although the conclusion about the nature of the interaction was similar to that formerly reported, that work revealed the role played by some technical aspects inherent to this type of modeling such as oscillatory behavior, size of the array of charges, finiteness of point charges, etc.

More recently, in an already mentioned paper, Damin et $a l .{ }^{20}$ reported on a study of the $\mathrm{CO} / \mathrm{MgO}(001)$ interaction
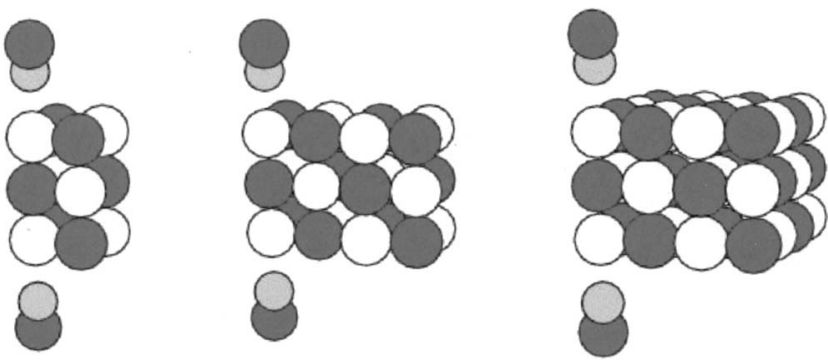

FIG. 1. Scheme of super cells for $\mathrm{CO} / \mathrm{MgO}(001)$ system at $\mathrm{CO}$ coverage of $\frac{1}{2}, \frac{1}{4}$, and $\frac{1}{8}$. Dark (red) circles, $\mathrm{O}$ atoms; gray circles, $\mathrm{C}$ atoms; white circles, $\mathrm{Mg}$ atoms.

using CRYSTAL $a b$ initio periodic calculations at both the HF and DFT levels using an alternative tool. Based on classical electrostatics, an expansion of the interaction energy was performed and compared with the results obtained for the $\mathrm{CO} / \mathrm{Na}^{+}$and $\mathrm{CO} / \mathrm{CO}_{2}$. These authors concluded that both polarization and charge transfer components play a negligible role in the binding process of $\mathrm{CO}$ to the surface. Likewise, the weak binding energy would result from a large cancellation between electrostatic and exchange repulsion components as formerly suggested by accurate embedded cluster calculations.

To test our CSOV implementation in CRYSTAL03 we have employed the same three-layer slab used in Ref. 20 to model the $\mathrm{MgO}(001)$ surface. The cell parameter used to build the slab is $4.250 \AA$, and some surface rippling have been included consisting of a displacement of both $\mathrm{Mg}^{2+}$ ions (inwards) and $\mathrm{O}^{2-}$ (outwards) by $0.001 \AA^{20}$ The carbon monoxide was perpendicularly adsorbed to the surface $\mathrm{Mg}$ atom through its $\mathrm{C}$ atom, and three different coverages were considered, namely, $\theta=\frac{1}{2}, \frac{1}{4}$, and $\frac{1}{8}$ (see Fig. 1). To keep as much symmetry as possible, the $\mathrm{CO}$ was deposited on both sides of the $\mathrm{MgO}(001)$ slab, after checking for the negligible intraslab $\mathrm{CO} / \mathrm{CO}$ interaction.

The calculations have been carried out at both the Hartree-Fock and the DFT levels of theory using Becke's three parameter hybrid exchange ${ }^{30}$ and the Lee-Yang Parr ${ }^{31}$ correlation functional (B3LYP). The level of accuracy in evaluating the Coulomb and exchange series is controlled by five parameters, ${ }^{18}$ for which standard values have been used $(6,6,6,6$, and 12). The DFT exchange-correlation contribution is evaluated by numerical integration over the cell volume. Here, integration was performed by means of a pruned grid with 75 radial and a maximum 434 angular points in the region relevant for chemical bonding (see keyword LGRID in the CRYSTAL manual ${ }^{18}$ ). The reciprocal space has been sampled according to a regular net with a shrinking factor of 4 , corresponding to six independent $k$ points in the irreducible Brillouin zone.

For the sake of coherence, the same basis sets considered in Ref. 20 have been used, namely, (I) and (II), referred to as A and B, respectively, in the original paper. Basis (I) is of Pople type with triple-zeta valence (TZV) quality plus polarization on atoms belonging to the $\mathrm{CO}$ molecule. The second basis set (II) is a modification of TZV of Schafer et al., ${ }^{32}$ which has been successfully used to model the interaction between $\mathrm{CO}$ and the complete series of alkaline cations with 
TABLE I. Binding energy (BE) and CSOV analysis (in $\mathrm{kJ} / \mathrm{mol}$ ) of $\mathrm{CO} / \mathrm{MgO}(001)$ interaction obtained from DFT and Hartree-Fock calculation at different coverages $\theta$ using basis sets (I) and (II). Contributions are labeled as follows: FO: electrostatic and Pauli repulsion; $\mathrm{CO}$-pol: $\mathrm{CO}$ polarization; $\mathrm{CO} \rightarrow$ Surf: charge transfer from $\mathrm{CO}$ towards the surface; Surf-pol: polarization of the surface; Surf $\rightarrow \mathrm{CO}$ : charge transfer from the surface towards the $\mathrm{CO}$ molecule.

\begin{tabular}{|c|c|c|c|c|c|c|c|c|}
\hline \multirow[b]{2}{*}{ Model } & \multirow[b]{2}{*}{$\theta$} & \multicolumn{6}{|c|}{ Contribution to the $\mathrm{BE}$} & \multirow[b]{2}{*}{$\mathrm{BE}^{\mathrm{a}}$} \\
\hline & & FO & CO-pol & $\mathrm{CO} \rightarrow$ Surf & Surf-pol & Surf $\rightarrow \mathrm{CO}$ & Sum & \\
\hline \multirow[t]{3}{*}{$\mathrm{B} 3 \mathrm{LYP} /(\mathrm{I})$} & \multirow{3}{*}{$\begin{array}{l}\frac{1}{2} \\
\frac{1}{4} \\
\frac{1}{8}\end{array}$} & -8.1 & 2.5 & 3.5 & 0.9 & 14.7 & 13.5 & 13.6 \\
\hline & & -7.8 & 2.5 & 3.7 & 0.8 & 14.7 & 13.9 & 14.0 \\
\hline & & -7.8 & 2.5 & 3.7 & 1.6 & 13.5 & 13.5 & 14.0 \\
\hline \multirow[t]{2}{*}{ B3LYP/(II) } & $\begin{array}{l}8 \\
\frac{1}{2}\end{array}$ & -11.8 & 3.0 & 3.5 & 1.7 & 12.4 & 8.8 & 9.1 \\
\hline & $\frac{1}{4}$ & -11.7 & 3.1 & 3.6 & 1.9 & 13.0 & 9.9 & 10.3 \\
\hline \multirow[t]{2}{*}{$\mathrm{HF} /(\mathrm{I})$} & $\frac{1}{2}$ & 0.6 & 0.4 & 0.8 & 0.1 & 3.6 & 5.5 & 6.2 \\
\hline & $\frac{1}{4}$ & 0.9 & 0.4 & 0.9 & 0.2 & 3.6 & 6.0 & 6.4 \\
\hline $\begin{array}{l}{\left[\mathrm{Mg}_{14} \mathrm{O}_{25}\right]^{22-}} \\
+\mathrm{TlPs}+\mathrm{PCs}^{\mathrm{b}}\end{array}$ & & -2.5 & 1.6 & 1.5 & 0.7 & 15.1 & 16.4 & \\
\hline
\end{tabular}

methods including electron correlation. ${ }^{33}$ A detailed description of both basis sets is provided in Ref. 20.

Concerning the localization of the $\mathrm{CO}$ molecule on the surface and its bond length, the optimized geometries reported in the same work ${ }^{20}$ have been considered for each basis set. Briefly, at the B3LYP level the distances between the $\mathrm{Mg}$ surface atom and the $\mathrm{C}$ atom of $\mathrm{CO}$ molecule are 2.61 and $2.64 \AA$, using basis set (I) and (II), respectively, while the distance estimated from Hartree-Fock calculations and basis set $\mathrm{A}$ is $3.0 \AA$ (with small variations depending on the coverage).

In the CSOV analysis the $\mathrm{MgO}$ (001) surface slab is ascribed to group $A$, and the set of almost noninteracting $\mathrm{CO}$ molecules to group $B$. Notice that all calculations were made using the same reciprocal space ( $k$ points) and space symmetry group. The computed wave functions vectors were used then to build the $\mathbf{T}^{(\mathbf{k}, r)}$ matrix at each $\mathbf{k}$ point and $r$-irreducible representation, and performed the SCF in constrained spaces. In this way, by conveniently setting to zero the elements of $\mathbf{F}^{\prime(\mathbf{k}, r)}$, a series of steps each associated to a given physical effects was carried out. A summary of results for each basis set, step by step, is gathered in Table I. In this Table the binding energy is defined as

$$
\mathrm{BE}=E(A)+E(B)-E(A B)
$$

where $E(A)$ is the energy of the $\mathrm{MgO}$ slab, $E(B)$ is the energy of the periodic array of adsorbed $\mathrm{CO}$ molecules without the underneath surface, and $E(A B)$ is the energy of the slab interacting with the periodic array of $\mathrm{CO}$ molecules. These quantities are negative, and a positive $\mathrm{BE}$ value corresponds to a bound state.

At the first glance one can observe a large difference between the DFT results and the HF ones in agreement with the analysis performed in Ref. 20 Some contributions to the interaction energy, as well as the BE itself computed from DFT calculations are roughly twice than estimated from HF wave functions. Furthermore, the FO contribution even changes sign. To rationalize these features lets start considering that at the FO step the energy contains two factors: the purely electrostatic contribution between the carbon monoxide molecule and the surface (attractive), and the Pauli repulsion (negative), which accounts for the interaction arising from the electronic densities of the fragments. On going from HF to DFT there is a shrinking of the orbitals that allow CO to lie closer to the surface by $\sim 0.4 \AA$. Such a shorter distance slightly increases the electrostatic attraction through interaction of $\mathrm{CO}$ with the surface field, but also largely enhances the Pauli repulsion. From this first step one can conclude that in DFT calculations the Pauli repulsion prevails over the electrostatic attraction, while is negligible at the HF level.

It is also worth to compare these values with that found using the $\left[\mathrm{Mg}_{14} \mathrm{O}_{25}\right]^{22-}$ cluster embedded in total ion potentials and point charges. ${ }^{11}$ As the basis set level in both cases is almost equivalent, let us focus our attention on the effect of the way one models the solid part. In the embedded cluster case, the FO step is also unfavorable likely due to the relatively short $\mathrm{CO}$ surface distance $(2.5 \AA)$ used in these calculations which increases the Pauli repulsion. However, the fact that the use of point charges leads to an overestimation of the pure electrostatic attraction has also to be considered. This put in evidence that, even in an ionic system, which is the case most favorable for using charge-embedding techniques, the periodic CSOV analysis can improve the description of the interactions occurring at the surface.

The three next steps account for the polarization of $\mathrm{CO}$, charge transfer from $\mathrm{CO}$ to the surface, and surface polarization. Concerning polarization, only the surface electrostatic field exerted on $\mathrm{CO}$ contribution has a significant (though very small) value, while surface polarization is negligible. Also significant is the contribution arising from the $\mathrm{CO}$ donation towards the surface. At the DFT level these values are similar to each other and noticeably larger than those found at the HF level. Finally, the last contribution, but not the least, results from the charge transfer from the surface towards the $\mathrm{CO}$ molecule. Since these values appear to dominate the interaction one would be inclined to ascribe to this physical ingredient as the main role. However, as previously 
discussed in Ref. 11 such large contribution is not physically meaningful and attributable (totally o partially) to the BSSE. This is in agreement with the corrected binding energy reported by Damin et al. ${ }^{20}$ where periodic boundary conditions were used to estimate the BSSE with the same basis set.

The sum of the contributions leads to final interaction energy close to the full BE estimation showing the coherence of the analysis and that most of the physical effects have been taken into account. The comparison of the present values with those reported in Ref. 11 does not qualitatively change the already described bond mechanism between $\mathrm{CO}$ and $\mathrm{MgO}$ surface in the sense that there is a weak interaction of mainly electrostatic origin with no noticeably chemical contributions. On the other hand, dispersion forces, not included neither in the DFT nor in the HF approaches, are predicted to bring only a low contribution. ${ }^{34}$ However, from a numerical point of view the coherence of the values reported in Table I supports the reliability of the present CSOV implementation, which was the focus of this work.

\section{CONCLUSIONS}

In this work we report the implementation of the CSOV method for the analysis of the interaction energy between two chemical entities under the periodic approximation. The routines for the analysis have been implemented in the $a b$ initio code CRYSTAL03. The method allows one to carry out the analysis both at the HF and DFT levels using the most popular exchange-correlation functionals. An initial numerical test for the $\mathrm{CO} / \mathrm{MgO}(001)$ system has been performed. The coherence of the different contributions, compared with those previously obtained using embedded cluster calculations shows the soundness of the implementation. The CSOV analysis here reported agrees with previous works and shows that there are no noticeable chemical contributions to the bond between the $\mathrm{CO}$ and the oxide surface. From the quantitative point of view, the main difference between the present periodic approach and previous CSOV analyses, performed on embedded clusters for this almost purely ionic system, arises from the weight attributed to the electrostatic forces between surface and adsorbate that seem to be slightly overestimated in the latter. Applications of this analysis to systems relevant in heterogeneous catalysis are currently ongoing.

\section{ACKNOWLEDGMENTS}

This work was funded by the Spanish Ministerio de Educación y Ciencia under Project No. MAT2005-1872, the Ramón y Cajal program [awarded to one of the authors (N.C.H.)], and the Mexican SEP-CONACYT (Project No. SEP-2004-CO1-46983). One of the authors (N.C.H.) thanks the Junta de Andalucía for supporting his stay at the UAEM. Unlimited CPU time on the IBMp690 32-processor supercomputer at UAEM through Project No. FOMES2000-SEP is gratefully acknowledged. The authors also thank Professor F. Illas and Professor A. Márquez for helpful discussions.

${ }^{1}$ P. S. Bagus, K. Hermann, and C. W. Bauschlicher, Jr, J. Chem. Phys. 80, 4378 (1984).

${ }^{2}$ P. S. Bagus, K. Hermann, and C. W. Bauschlicher, Jr, J. Chem. Phys. 81, 1966 (1984).

${ }^{3}$ K. Morokuma, J. Chem. Phys. 55, 1236 (1971).

${ }^{4}$ T. Ziegler and A. Rauk, Theor. Chim. Acta 46, 1 (1977).

${ }^{5}$ P. S. Bagus and F. Illas, J. Chem. Phys. 96, 8962 (1992).

${ }^{6}$ C. W. Bauschlicher, P. S. Bagus, C. J. Nelin, and B. O. Ross, J. Chem. Phys. 85, 354 (1986).

${ }^{7}$ K. Albert, K. M. Neyman, V. A. Nasluzov, S. Ph. Ruzankin, C. Yeretzian, and N. Rösch, Chem. Phys. Lett. 245, 671 (1995).

${ }^{8}$ K. M. Neyman, P. Strodel, S. Ph. Ruzankin, N. Schlensog, H. Knoezinger, and N. Rösch, Catal. Lett. 31, 273 (1995).

${ }^{9}$ M. Dupuis, A. M. Márquez, and E. R. Davidson, HOND095.3, Quantum Chemistry Program Exchange (QCPE); Bloomington, Indiana (CSOV implementation by A. M. Márquez).

${ }^{10}$ A. M. Márquez, N. López, M. García-Hernández, and F. Illas, Surf. Sci. 442, 463 (1999).

${ }^{11}$ J. A. Mejías, A. M. Márquez, J. F. Sanz, M. Fernández-García, J. M. Ricart, C. Sousa, and F. Illas, Surf. Sci. 327, 59 (1995).

${ }^{12}$ J. F. Sanz, N. C. Hernández, and A. Márquez, Theor. Chem. Acc. 104, 317 (2000).

${ }^{13}$ C. Pisani, R. Dovesi, and C. Roetti, Hartree-Fock Ab Initio Treatment of Crystalline Solids (Springer-Verlag, Berlin, 1988).

${ }^{14}$ M. C. Payne, M. P. Teter, D. C. Allan, T. A. Arias, and J. D. Joannopoulos, Rev. Mod. Phys. 64, 1045 (1992).

${ }^{15}$ N. Cruz Hernández, Jesús Graciani, A. Márquez, and J. F. Sanz, Surf. Sci. 575, 189 (2005).

${ }^{16}$ J. R. B. Gomes, F. Illas, N. Cruz Hernández, A. Márquez, and J. F. Sanz, Phys. Rev. B 65, 125414 (2002).

${ }^{17}$ J. Oviedo and J. F. Sanz, Surf. Sci. 397, 23 (1998).

${ }^{18}$ V. R. Saunders, R. Dovesi, C. Roetti, R. Orlando, C. M. Zicovich-Wilson, N. M. Harrison, K. Doll, B. Civalleri, I. J. Bush, P. D. Arco, and M. Llunell, CRYSTAL03 User's Manual (Università di Torino, Turin, 2003); URL http://www.crystal.unito.it

${ }^{19}$ J. Sauer, P. Ugliengo, E. Garrone, and V. R. Saunders, Chem. Rev. (Washington, D.C.) 94, 2095 (1994).

${ }^{20}$ A. Damin, R. Dovesi, A. Zecchina, and P. Ugliengo, Surf. Sci. 479, 255 (2001).

${ }^{21}$ F. Illas, G. Pacchioni, A. Pelmenschikov, L. G. M. Pettersson, R. Dovesi, C. Pisani, K. M. Neyman, and N. Rösch, Chem. Phys. Lett. 306, 202 (1999).

${ }^{22}$ C. Pisani, R. Dovesi, and C. Roetti, Hartree-Fock Ab Initio Treatment of Crystalline Solids, Lecture Notes in Chemistry Vol. 48 (Springer-Verlag, Berlin, 1988).

${ }^{23}$ C. M. Zicovich-Wilson and R. Dovesi, Int. J. Quantum Chem. 67, 299 (1998); Int. J. Quantum Chem. 67, 311 (1998).

${ }^{24}$ H. J. Monkhorst, and J. D. Pack, Phys. Rev. B 13, 5188 (1976).

${ }^{25}$ This particular aspect of the method is not actually mentioned in the previous literature on CSOV implementations. F. Illas (private communication).

${ }^{26}$ E. A. Colbourn, Surf. Sci. Rep. 15, 281 (1992).

${ }^{27}$ H.-J. Freund, H. K. Kuhlenbeck, and V. Staemmler, Rep. Prog. Phys. 59, 283 (1996).

${ }^{28}$ R. Wichtendahl, M. Rodriquez-Rodrigo, U. Härtel, H. Kuhlenbeck, and H.-J. Freund, Surf. Sci. 423, 90 (1999).

${ }^{29}$ G. Pacchioni, G. Cogliandro, and P. S. Bagus, Int. J. Quantum Chem. 42, 1115 (1992)

${ }^{30}$ A. D. Becke, J. Chem. Phys. 98, 5648 (1993).

${ }^{31}$ C. Lee, W. Yang, and R. G. Parr, Phys. Rev. B 37, 785 (1988).

${ }^{32}$ A. Schafer, C. Huber, and R. Ahlrichs, J. Chem. Phys. 100, 5829 (1994).

${ }^{33}$ A. M. Ferrari, P. Ugliengo, and E. Garrone, J. Chem. Phys. 105, 4129 (1996).

${ }^{34}$ M. A. Nygren, L. G. M. Petterson, Z. Barandiaran, and L. Seijo, J. Chem. Phys. 100, 2010 (1994). 